\title{
Transcriptome Comparison of Rhizome and Leaf of Rhizome of Ligusticum Chuanxiong on the Effects of Active Metabolites
}

\section{Xianhong HE}

Chengdu Medical College

Qing HAO

Southwest Medical University

Zhengxue cai

Chengdu Medical College

Gaolei Li

Zhejiang Gongshang University

\section{Ping Wei}

Sichuan Academy of Chinese Medicine Sciences

\section{Zhonglin Zhang}

Chengdu Medical College

\section{Zhixiang Yan}

Sichuan Academy of Chinese Medicine Sciences

Yi-guan ZHANG ( $\square$ yiguanzhang@126.com )

Southwest Medical University https://orcid.org/0000-0002-0608-1863

Jun-ning ZHAO

Southwest Medical University

\section{Research}

Keywords: Ligusticum Chuanxiong, transcriptome, RNA sequences, secondary metabolism

Posted Date: February 17th, 2021

DOI: https://doi.org/10.21203/rs.3.rs-203524/v1

License: (c) (1) This work is licensed under a Creative Commons Attribution 4.0 International License. Read Full License 


\section{Abstract}

\section{Background}

The rhizome of Ligusticum chuanxiong is a well-known traditional Chinese medicine. The major bioactive compounds found in Ligusticum chuanxiong are Ferulic Acid with 4- hydroxyl -3- methoxybenzene acrylic acid. However, the epigenetic mechanisms and biosynthetic pathways of active metabolites based on the transcriptomic analysis of Ligusticum chuanxiong rhizome have remained unclear. To clarify the key genes in rhizome formation and active metabolite synthesis pathways of Rhizome of Ligusticum chuanxiong, we focus on the change between the investigate the rhizome and leaf of Ligusticum chuanxiong through transcriptomic analysis.

Methods

The RNA extracted from rhizomes and leaves of Ligusticum chuanxiong were sequenced using the Illumina platform, yielding 295,725 unique transcripts with N50 of 778 bp. The transcripts against annotation databases including GO (Gene Ontology) and KEGG (Kyoto Encyclopedia of Genes and Genomes) to identify differentially expressed genes (DEGs).

Results

ystematic analysis of transcriptome profiles revealed that a total of 1707 differentially expressed unigenes were found in the two tissues, among which 1246 were down-regulated and 462 were upregulated. Remarkably, some DEGs are enriched in the biosynthetic pathway of phenylalanine ammonia lyase, one of the key enzymes in the pathway of ferulic acid synthesis.

\section{Conclusions}

Through the transcriptome analysis, we have identified the regulatory mechanisms and epigenetic influences in the secondary metabolic biosynthetic pathway of Ligusticum chuanxiong. Among them, DEGs analysis revealed ferulic acid and other secondary metabolites are some of the major products in the biosynthetic pathway of Ligusticum chuanxiong.

\section{Background}

Ligusticum chuanxiong (called Chuanxiong, CX in Chinese), the dried rhizome of Ligusticum Chuanxiong Hort, is mainly distributed in Sichuan and cultivated and bred in Yunnan, Guizhou, Guangxi and other regions $^{[1,2]}$. It shows an ideal therapeutic effect on cardiovascular and cerebrovascular diseases, and is also used as a preventive medicine to supply the vitamins necessary to maintain fitness ${ }^{[3]}$. In clinical practice, Ligusticum chuanxiong is widely used to treat thromboembolism heart diseases ${ }^{[4]}$, headache ${ }^{[5}$, ${ }^{6]}$, wind-damp ${ }^{[7]}$. Recent studies have found that the active metabolites of Ligusticum chuanxiong including ligustrazine ${ }^{[8]}$, ferulic acid ${ }^{[9]}$, ligustilide ${ }^{[10]}$, polysaccharide and the others ${ }^{[11]}$. Ferulic acid, a kind 
of phenolic acid, is one of the most important active ingredients in Ligusticum chuanxiong ${ }^{[12]}$. Previous studies provided that ferulic acid has strong antioxidant properties and has a good function scavenge hydrogen peroxide, hydroxyl radicals, superoxide radicals and peroxynitrite ${ }^{[13]}$. In terms of the application of disease treatment, ferulic acid has antibacterial and anti-inflammatory properties ${ }^{[9]}$, which can prevent coronary heart disease $\mathrm{e}^{[14]}$, protect ovaries ${ }^{[15]}$ and inhibit liver damage $\mathrm{e}^{[16]}$, and even play a role in cancer prevention ${ }^{[17,18]}$. Due to the special therapeutic effects of ferulic acid ${ }^{[19]}$, much previous research has been done on the organic synthesis of ferulic acid, and a large number of ferulic acid derivatives have been synthesized and demonstrated their activity ${ }^{[20,21]}$.

However, there are few reports on the interaction between genetic information and functional phenotypes based on transcriptome data analysis of Ligusticum chuanxiong. With the development of the Nextgeneration sequencing technology ${ }^{[22]}$, whole transcriptome sequencing plays a crucial role in deciphering genome structure and function, identifying genetic networks underlying cellular, physiological, biochemical and biological systems and establishing molecular biomarkers that respond to diseases, pathogens and environmental challenges ${ }^{[23]}$. Unlike the genome, the transcriptome is data that shows the expression level of all genes in a specific organ, tissue or cell at a specific stage of development. Thus, it is especially suitable for the discovery and identification of genes related to the medicinal function of plants and the study of the regulation mechanism of gene expression ${ }^{[24]}$.

In our study, we collected the rhizome and leaf tissues of Ligusticum chuanxiong and extracted the total RNA. We used HiSeq2000 high-throughput sequencing technology to conduct whole transcriptome sequencing on the leaf and rhizome of Ligusticum chuanxiong, and a total of $25 \mathrm{~Gb}$ of raw data was obtained. The clean reads after filtering were assembled and annotated against public databases to assess their differences in gene function of Ligusticum chuanxiong. Furthermore, we provided a perspective on the genetic intervention strategy and molecular mechanism of Ligusticum chuanxiong.

\section{Materials And Methods}

\section{Plant materials}

Ligusticum chuanxiong was collected from a cultivating in Pengzhou, Sichuan province, China. The leaf and rhizomes of Ligusticum chuanxiong were labeled, cleaned and stored in cryostat tubes, sealed and packed in liquid nitrogen, transported to the laboratory and stored at $-80^{\circ} \mathrm{C}$ in the refrigerator for RNA extraction. Each experiment was repeated three times (figure 1).

\section{RNA extraction}

Precisely weigh $1 \mathrm{~g}$ of rhizome and leaf tissue of Ligusticum chuanxiong, ground into powder with liquid nitrogen, and extract total RNA according to TRIzol protoco ${ }^{[25]}$. RNA quality and integrity were assessed using the Nanodrop based on the absorbance ratios at 260/280 and 260/230 numbers greater than 2.0. 
All samples selected for sequencing had an RNA integrity number over 8 . Total RNA was restored in the refrigerator at the temperature of $-80^{\circ} \mathrm{C}$. Each experiment was repeated three times.

\section{RNA sequencing}

Enrichment of poly $(\mathrm{A})$ mRNA using magnetic beads with Oligo(dT) $\triangle$ Synthesize cDNA strands to construct a library. The prepared library was sequenced on the Illumina platform to obtain the raw reads. The raw reads were cleaned by removing adaptor sequences and the low-quality reads (more than $40 \%$ bases with Q-value $<30$ or more than $5 \% \mathrm{Ns}$ in the read), which is performed by fastp (version 0.20 .0 ).

\section{Transcriptome de novo assembly}

The clean reads were then assembled using Trinity (v2.9.1). The longest transcript of each cluster was regarded as the unigenes for further downstream analysis. The TransDecoder (version 5.5.0) was used to predict the ORFs for differentially expressed gene analysis, working with the unigene mentioned previously. The quality and completeness of assembly were assessed by BUSCO (version 3.0.1). The databases virdiplantae_odb10 and embryophyta_odb10 were used as the lineage-specific dataset. Identification of the microsatellite loci was performed by perl script misa (version 2.0).

\section{Transcriptome functional annotation}

To predict putative gene function, genes were annotated by online functional annotation tool eggNOGmapper, which is a web-tool for fast functional annotation of novel sequence, related to KEGG, GO and other databases. The result of eggNOG-mapper was parsed by homemade scripts. The R package cluster Profiler (version 3.16.0) was used for enrichment clustering.

\section{Differentially expressed genes (DEGs)}

Relative gene expression levels of each unigene were determined by calculating the sum of the reads mapping to each contig, and then normalized by converting the reads counts to (reads per kilobase per million reads, or RPKM) across the transcriptome. The DEGs were obtained by using R package edgeR (version 3.30.3). A gene was considered as DEG if its expression level in one tissue was significantly higher than that in the other based on the Fold Change $(F C)$ ratio $>=3$ and $p<0.01$. All the data were corrected according to the Benjamini-Hochberg formula.

\section{Data Records}

The filtered original RNA sequencing reads have been deposited at the NCBI Sequence Read Archive under the BioProject study accession PRJNA672790 
(https://www.ncbi.nlm.nih.gov/bioproject/PRJNA672790). Functional annotation of the Trinity transcriptome assembly is available as a supplementary gff file at figshare (Functional annotation of the Trinity transcriptome assembly). Summary of the microsatellite loci identified by the MISA tool in the Trinity assembly based on the filtered reads is available as a supplementary excel file at figshare (Summary of the microsatellite loci identified by the MISA tool in the Trinity assembly based on the filtered reads). PCR primers designed for these microsatellite loci using Primer3 online tool are available as a supplementary excel file at figshare (PCR primers designed for the microsatellite loci identified by the MISA tool in the Trinity assembly based on the filtered reads using Primer3 online tool).

\section{Results}

\section{Quality control and assemblies}

Two cDNA libraries were sequenced using Illumina technology, respectively. A total of $25 \mathrm{~Gb}$ was obtained with Q20 of $97.34 \%$ and Q30 of $90.49 \%$. After removing adaptor sequences and the low-quality reads, $119,604,818$ and $126,274,844$ clean reads were generated from the rhizome and leaf, respectively. Then all clean reads were pooled up together for Trinity assembly. A total of 295,725 transcripts (N50 length 778) were generated and the longest transcripts in its cluster were treated as unigenes, which got 248,454 unigenes (N50 length 468). Trans Decoder software was used to identify possible coding regions within Trinity transcriptome assemblies, which got 72,363 (N50 length 1041) coding regions.

\section{Functional annotation}

Both of them were uploaded to eggNOG website to perform function annotation. From the perspective of annotating functional proteins, the sequence which treats the longest transcripts in its cluster as unigenes, only 17762 sequences hit eggNOG database. By contrast, the predicted coding sequencing performed better, hit 61448 sequences in the database. The followed analysis is based on the latter result.

\section{Differential expression gene analysis}

According to the definition of DEGs, there were a totally of 1,708 unigenes expressed differently between two tissue, 1,246 of which were down-regulated and 462 unigenes were up-regulated. The first ten differentially expressed genes were labeled (figure 2). In order to find the most important key genes related to the synthesis of active metabolites of Ligusticum chuanxiong, the top ten up- and downregulated differentially expressed genes were marked in the volcano map. It is very interesting to note that among the up-regulated genes, there are three genes with the most significant differences, including two Cupin (DN72188_c1_g1_i1, DN72188_c1_g1_i2) and a BURP domain-containing protein (DN99636_c2_g1_i1). The Cupin family is a family of multi-functional proteins within which several allergic proteins have been identified. However, most of the cupin allergenic proteins are pea globulin and 
soy globulin. BURP domain-containing protein is a plant-specific family of proteins with multiple protein functions involving in the development and expression in embryogenesis. In the three most significant down-regulated genes, there were two Ribulose bisphosphate carboxylase activase (RCA) (DN73345_c0_g1_i2, DN73345_c0_g1_i1) and an Involved in Biosynthesis of the Thiamine precursor Thiazole (DN63265_c0_g1_i2). RCA is a Protein that binds adenosine 5'-triphosphate (ATP), ribonucleotide adenosine that carries three phosphate groups esterified to the sugar moiety. It is the cell's source of energy and phosphate.

\section{Gene Ontology analysis}

Most of the annotated DEGs were enriched in chloroplasts and photosynthesis, which was due to tissue specificity. Notably, some of the DEGs were enriched in biological processes, biosynthesis, IGE affinity showing the highest enrichment, followed by glutathione transferase activity, immunoglobulin affinity, IGE affinity and immunoglobulin affinity all showing very high enrichment, which is related to some storage proteins produced by plants that can induce high immunity. Allergy is one of the causes of autoimmune reactions, therefore, the immune-related side effects in the application of Ligusticum chuanxiong should also be taken into consideration. Among them, it is worth noting that the activity of phenylalanine aminolytic enzyme, one of the important synthetic enzymes in the ferulic acid synthesis pathway, shows a relatively high enrichment (Figure 3). In terms of molecular function, monooxygenase and oxidoreductase activities are the most prominent. Plasmodial spheres, vesicle-like cavities and chloroplast membranes are the major cellular components.

\section{Clusters of Orthologous Groups analysis}

Overall, 1593 DEGs were assigned to COG classifications (Figure 4). Regardless of the most function unknown category $(448,28.1 \%)$, the following categories were posttranslational modification, protein turnover, chaperones $(136,8.53 \%)$, carbohydrate transport and metabolism $(126,7.90 \%)$, signal transduction mechanisms $(1.14,7.16 \%)$, secondary metabolites biosynthesis, transport and catabolism $(95,5.96 \%)$, energy production and conversion (88,5.52\%). Please see the attachment for the rest categories (Supplementary Table S2). Most of the genes in the COG database are genes of unknown function, and the role of these genes in plant biosynthetic and metabolic networks is still unknown to us. What interests us is that some genes were enriched in categories where posttranslational modifications, protein turnover, chaperones showed high enrichment in categories, and these proteins are essential biological processes for the completion of life activities in chuanxiong rhizome. We also found a high enrichment of carbohydrate transport and metabolism and secondary metabolites biosynthesis, transport and catabolism, which is consistent with the fact that chuanxiong produces a variety of active metabolites and Storage of nutrients is closely related.

\section{KEGG pathways analysis}


To explore the specification of biological pathways which were active in the root and leaf of $\mathrm{L}$.

chuanxiong, 497 DEGs were assigned to KEGG pathways (figure 5). The top 14 pathways with the highest enrichment factor were shown in KEGG enrichment analysis. The most representative pathway where DEGs enriched is phenylpropanoid biosynthesis[ko00940] (37, 7.44\%), photosynthesis[ko00195] (27, $5.43 \%)$, glycolysis/gluconeogenesis[ko00010] $(25,5.03 \%)$, carbon fixation in photosynthetic organisms[ko00710] $(24,4.82 \%)$, starch and sucrose metabolism[ko00500] $(24,4.82 \%)$, glyoxylate and dicarboxylate metabolism[ko00630] (22,4.43\%), porphyrin and chlorophyll metabolism[ko00860] (20, $4.02 \%)$, tryptophan metabolism[ko00380] (18,3.62\%). And also, the pathway about Sesquiterpenoid and triterpenoid biosynthesis[ko00909] (10, 2.01\%) were enriched. The phenylpropanoid biosynthesis was the most diverse and highly enriched among the KEGG pathway, which may be due to the tissue specificity of Ligusticum chuanxiong. In addition, phenylpropanoid biosynthesis was the most differentially enriched pathway in the KEGG pathway, which may be due to the tissue specificity of Ligusticum chuanxiong. Phenylpropanoids have been shown to be an important intermediate in the pathway of the synthesis of the active metabolite ferulic acid of Ligusticum chuanxiong.

\section{BUSCO analysis}

To test the transcriptome assemblies for completeness, a survey on the conserved orthologous genes was done using BUSCO (Table 1).

\section{Microsatellite discovery and testing}

The microsatellite discovery of the unigenes sequences found 3394 microsatellite loci in 72,363 sequences. (Supplementary Table S1) Among them, 499 SSRs represented microsatellite loci with mononucleotide motifs, 966-dinucleotide, 1,891-trinucleotide, 20-tetranucleotide, 5-pentanucleotide, and 13-hexanucleotide motifs. As for the frequency of the classified repeat types, A/T represents mononucleotide motif, AG/CT represents dinucleotide motif, ATC/ATG represents trinucleotide motif, followed by AAG/CTT. From the analysis of the composition base types of the repeat units, a total of 34 different composition repeat units were found, including 2 mononucleotide repeats, 4 dinucleotide repeats, 10 trinucleotide repeats, 5 tetranucleotide repeats, 5 pentanucleotide repeats, and 8 hexanucleotide repeats. For frequency of classified repeat types, $A / T$ represents mononucleotide motif, AG/CT represents dinucleotide motif, ATC/ATG represents trinucleotide motif, followed by AAG/CTT. Among mononucleotides $\mathrm{G} / \mathrm{C}$ is less $(32,0.94 \%)$ and $\mathrm{A} / \mathrm{T}$ is most $(467,13.76)$; among dinucleotides CG/CG is least $(5,0.15 \%)$ and $A G / C T$ is most $(697,20.54 \%)$ and most in total nucleotide repeats; among trinucleotides ACG/CGT repeats are least $(25,0.74 \%)$. ATC/GAT repeats were the most frequent (428, $12.61 \%$ ); tetranucleotide, pentanucleotide, and hexanucleotide numbers each had fewer repeated units, with ACAT/ATGT repeats most frequent in tetranucleotides $(13,0.38)$, less frequent in pentanucleotides, and ACTCAT/AGTATG repeats most frequent in hexanucleotides (3, 0.09\%).

\section{Discussion}


The rhizome of Chuanxiong is used for its mature rhizome, which is mainly used for promoting blood circulation, dispelling wind and relieving pain ${ }^{[26]}$. The quality and content of chuanxiong rhizome grown in different regions have been found to be different, and the content of active ingredients of different plants in the same region has great variability and instability with the pharmacological effects and efficacy of chuanxiong rhizome ${ }^{[27]}$. Due to the importance of chuanxiong rhizome in traditional Chinese medicine, there have been increasing attempts to understand its physiological properties, epigenetic and molecular mechanisms. Therefore, we constructed whole transcriptomes of rhizomes and leaves of Ligusticum chuanxiong and investigated the mechanisms of its genetic properties and metabolites.

In this study, we collected tissues from the rhizomes and leaf of Chuanxiong rhizome in Pengzhou region for transcriptome sequencing. Functional annotation and differentially expressed genes (DEGs) analysis were mainly performed from the sequencing results. In the DEGs of leaf and roots of Ligusticum chuanxiong, we found that most of the DEGs were enriched in chloroplasts and photosynthesis, which was shown by data comparison to being due to tissue specificity. It is worth noting that the enrichment of DEGs class to a biological process, biosynthesis, IGE affinity presented the highest concentration, the second is the activity of glutathione transferase, immunoglobulin affinity, IGE affinity and immunoglobulin affinity are showing very high concentration, which related to plant to produce some storage proteins, these proteins can cause high immune reaction, such as allergy is one of the reasons. Therefore, we suggest that some immune-related side effects should also be paid attention to in the application of Chuanxiong. In the results of GO database analysis, there is a high enrichment of phenylalanine aminolytic enzyme in the biological processes, and the production of ferulic acid is closely related to phenylalanine aminolytic enzyme. Phenylalanine ammonia lyase catalyzes an important ratelimiting step ${ }^{[28]}$ in the phenylalanine pathway, providing precursors such as cinnamic acid, vanillin acid and ferulic acid to a variety of secondary metabolites including bitter glycosides ${ }^{[29]}$. The most important active component of Chuanxiong is ferulic acid ${ }^{[30]}$, which is used by the Chinese Pharmacopoeia as the main indicator for the quality evaluation of these herbs ${ }^{[31]}$.

There are two main biological sub-pathways related to ferulic acid metabolism: one is the synthesis of caffeic acid and ferulic acid from phenylalanine via PAL and cinnamate-4-hydroxylase $(\mathrm{C} 4 \mathrm{H})$, and ferulic acid can be metabolized to 5-hydroxyferulic acid via ferulic acid 5-hydroxylase $(\mathrm{F} 5 \mathrm{H})^{[32]}$; the second is the synthesis of caffeic acid and ferulic acid via 4-coumaroyl-coenzyme (4-Coumaroyl-coenzyme); and the second is the metabolism of ferulic acid via 4-Coumaroyl-coenzyme (4-Coumaroyl-coenzyme) ${ }^{[33,34]}$. The first pathway can generate active substances such as caffeic acid and ferulic acid, which have antiinflammatory and immunomodulatory effects, and ferulic acid also has antithrombotic effects; the second pathway can generate non-pharmacological active substances such as pineal and lignin. Therefore, by inhibiting ferulic acid decomposing enzyme downstream of PB pathway through biomolecular technology, ferulic acid can be accumulated in excess without increasing the content of lignin (ash), thus enhancing the medicinal value of Ligusticum Chuanxiong. The key enzyme genes of the ferulic acid synthesis pathway were identified by transcriptome analysis, including four PAL genes and one cinnamoyl-coenzyme A-reductase (CCR) gene, two BDP genes, three late embryonic developmental 
enrichment protein genes and one Rop guanine nucleotide exchange factor gene, which are related to the formation of rhizome. These genes will be potential target genes for our future transgenic engineering or genetic modification of the herbal quality of Ligusticum chuanxiong.

In recent years, with the development of high-throughput sequencing technology and the reduction of the cost of second-generation sequencing as well as the update of SSR development software, SSRs for medicinal plants have been developed and applied ${ }^{[35]}$. Since SSRs have interspecific variability, we were able to use some high-frequency SSRs as biomarkers for interspecific kinship analysis of Ligusticum chuanxiong. We analyzed simple repeat sequences in chuanxiong and found 499 SSRs represented microsatellite loci with mononucleotide motifs. The trinucleotide repeats of Ligusticum chuanxiong SSR were dominated by ATC/GAT and AAG/CTT, which were basically consistent with the trinucleotide repeat characteristics of Salvia miltiorrhiza ${ }^{[36]}$. Therefore, SSR molecular markers are of great value in the study of the genetic diversity of Ligusticum chuanxiong.

\section{Conclusion}

In conclusion, we constructed a high-quality transcriptome of rhizome and leaf of Ligusticum Chuanxiong using the Illumina sequencing platform. By comparing transcriptome analyses, we identified candidate genes in these DEGs related to the active metabolite of chuanxiong rhizome, ferulic acid, for phenylalanine aminolytic enzymes as well as terpene synthesis and some other secondary metabolites. These candidate genes can provide a basis for future studies of the molecular mechanisms of rhizome formation, development and secondary metabolism, and will be useful in future clinical applications in Chinese medicine.

\section{Abbreviations}

GO. Gene Ontology; KEGG. Kyoto Encyclopedia of Genes and Genomes; DEGs. Differentially expressed genes. RCA. Ribulose bisphosphate carboxylase activase; SSRs. Simple sequence repeats;

The authors declare no competing interests. PAL. Phenylalanine ammonia lyase catalyzes;

\section{Declarations}

\section{Acknowledgements}

We greatly appreciate Dr. Y. D. Li (Zhejiang Gongshang Univeristy, China) for providing help.

\section{Author contributions}

Author contributions Q.H. and X.H.H. conceived and designed the experiments and the analytical strategy. J.N.Z prepared plant samples, conducted experiments and performed laboratory work. Q.H. and G.L.L. 
performed data analysis. P.W and Y.G.Z advised and supervised the project. X.H.H. and Q.H. drafted the manuscript. All authors provided feedback on the draft manuscript and approved the final manuscript for submission.

\section{Fundding}

National Basic Research Program of China (973 Program) (2009CB522801); National Science and Technology Major Projects for "Major New Drugs Innovation and Development"(2011ZX09401-304, 2015ZX09501004-001-005); National Natural Science Foundation of China (30672651, 81073047, 81470180); Sichuan Traditional Chinese Medicine Administration Project(20017Z001)

\section{Availability of data and materials}

The datasets supporting the conclusions of this article are included within the article and its additional fles.

\section{Ethics approval and consent to participate}

Not applicable.

\section{Consent for publication}

Not applicable.

\section{Competing interests}

The authors declare no competing interests.

\section{Author details}

${ }^{1}$ College of Pharmaceutical Sciences, Southwest Medical University, Luzhou, 646000, China.

${ }^{2}$ College of Pharmaceutical Sciences, Chengdu Medical College, Chengdu,610500, China.

${ }^{3}$ Sichuan Center for Translational Medicine of Traditional Chinese Medicine, Institute of Translational Pharmacology, Sichuan Academy of Chinese Medicine Sciences, Chengdu, 610042, China.

${ }^{4}$ Department of Biological Engineering, School of Food Science and Biotechnology, Zhejiang Gongshang Univeristy, Hangzhou, 310018, China 


\section{References}

1. Chen, Z., et al., A systematic review on the rhizome of Ligusticum chuanxiong Hort. (Chuanxiong). Food Chem Toxicol, 2018. 119: p. 309-325.

2. Li, W., et al., Advances in the chemical analysis and biological activities of chuanxiong. Molecules, 2012. 17(9): p. 10614-51.

3. Yang, X., X. Zeng, and T. Wu, WITHDRAWN: Chuanxiong preparations for preventing stroke. Cochrane Database Syst Rev, 2016(6): p. CD006765.

4. Li, $\mathrm{H}$., et al., The effects of Chuanxiong on the pharmacokinetics of warfarin in rats after biliary drainage. J Ethnopharmacol, 2016. 193: p. 117-124.

5. Wang, Y., et al., A Chinese Prescription Chuanxiong Chatiao San for Migraine: A Systematic Review and Meta-Analysis of Randomized Controlled Trials. Evid Based Complement Alternat Med, 2019. 2019: p. 2301680.

6. Mi, Y., et al., Pharmacokinetic comparative study of GAS with different concentration of tetramethylpyrazine and ferulic acid on liver-yang hyperactivity migraine model by blood-brain microdialysis method. J Pharm Biomed Anal, 2020. 191: p. 113643.

7. Wang, M., et al., Ligusticum chuanxiong exerts neuroprotection by promoting adult neurogenesis and inhibiting inflammation in the hippocampus of ME cerebral ischemia rats. J Ethnopharmacol, 2020. 249: p. 112385.

8. Zhang, F., et al., Ligand Activation of PPARgamma by Ligustrazine Suppresses Pericyte Functions of Hepatic Stellate Cells via SMRT-Mediated Transrepression of HIF-1alpha. Theranostics, 2018. 8(3): p. 610-626.

9. Liu, Y.M., et al., Ferulic acid inhibits neuro-inflammation in mice exposed to chronic unpredictable mild stress. Int Immunopharmacol, 2017. 45: p. 128-134.

10. Long, F.Y., et al., Klotho upregulation contributes to the neuroprotection of ligustilide against cerebral ischemic injury in mice. Eur J Pharmacol, 2018. 820: p. 198-205.

11. Huang, C., et al., A pectic polysaccharide from Ligusticum chuanxiong promotes intestine antioxidant defense in aged mice. Carbohydr Polym, 2017. 174: p. 915-922.

12. Chaudhary, A., et al., Ferulic Acid: A Promising Therapeutic Phytochemical and Recent Patents Advances. Recent Pat Inflamm Allergy Drug Discov, 2019. 13(2): p. 115-123.

13. Zdunska, K., et al., Antioxidant Properties of Ferulic Acid and Its Possible Application. Skin Pharmacol Physiol, 2018. 31(6): p. 332-336.

14. Liu, Z., et al., Ferulic acid increases intestinal Lactobacillus and improves cardiac function in TAC mice. Biomed Pharmacother, 2019. 120: p. 109482.

15. Macias-Cruz, U., et al., Effects of dietary ferulic acid on reproductive function and metabolism of prepubertal hairbreed ewes during the anestrous season. Theriogenology, 2018. 119: p. 220-224.

16. Mu, M., et al., Ferulic acid attenuates liver fibrosis and hepatic stellate cell activation via inhibition of TGF-beta/Smad signaling pathway. Drug Des Devel Ther, 2018. 12: p. 4107-4115. 
17. Maruyama, H., et al., Biochemical Characterization of Ferulic Acid and Caffeic Acid Which Effectively Inhibit Melanin Synthesis via Different Mechanisms in B16 Melanoma Cells. Biol Pharm Bull, 2018. 41(5): p. 806-810.

18. Cui, D., et al., Synthesis, characterization and antitumor properties of selenium nanoparticles coupling with ferulic acid. Mater Sci Eng C Mater Biol Appl, 2018. 90: p. 104-112.

19. Mancuso, C. and R. Santangelo, Ferulic acid: pharmacological and toxicological aspects. Food Chem Toxicol, 2014. 65: p. 185-95.

20. Klepacka, J. and L. Fornal, Ferulic acid and its position among the phenolic compounds of wheat. Crit Rev Food Sci Nutr, 2006. 46(8): p. 639-47.

21. Zhang, X., et al., Bioactive thionic compounds and aromatic glycosides from Ligusticum chuanxiong. Acta Pharm Sin B, 2018. 8(5): p. 818-824.

22. Levy, S.E. and R.M. Myers, Advancements in Next-Generation Sequencing. Annu Rev Genomics Hum Genet, 2016. 17: p. 95-115.

23. Deng, S., et al., De novo transcriptome sequencing and gene expression profiling of Magnolia wufengensis in response to cold stress. BMC Plant Biol, 2019. 19(1): p. 321.

24. van Dijk, E.L., et al., The Third Revolution in Sequencing Technology. Trends Genet, 2018. 34(9): p. 666-681.

25. Rio, D.C., et al., Purification of RNA using TRIzol (TRI reagent). Cold Spring Harb Protoc, 2010. 2010(6): p. pdb prot5439.

26. Hu, J., et al., Ultrasonic extraction, antioxidant and anticancer activities of novel polysaccharides from Chuanxiong rhizome. Int J Biol Macromol, 2016. 85: p. 277-84.

27. Zou, J., et al., Recent progress in the structural modification and pharmacological activities of ligustrazine derivatives. Eur J Med Chem, 2018. 147: p. 150-162.

28. Zhang, X., et al., Ferulic acid derivatives from Ligusticum chuanxiong. Fitoterapia, 2018. 125: p. 147154.

29. Bhat, W.W., et al., A phenylalanine ammonia-lyase ortholog (PKPAL1) from Picrorhiza kurrooa Royle ex. Benth: molecular cloning, promoter analysis and response to biotic and abiotic elicitors. Gene, 2014. 547(2): p. 245-56.

30. Liang, X.L., et al., Quantitative Evaluation of the Mechanism Underlying the Biotransportation of the Active Ingredients in Puerariae lobatae Radix and Chuanxiong rhizoma. Phytother Res, 2015. 29(9): p. 1396-1403.

31. Guo, L., et al., Identification and quantification of the quality markers and anti-migraine active components in Chuanxiong Rhizoma and Cyperi Rhizoma herbal pair based on chemometric analysis between chemical constituents and pharmacological effects. J Ethnopharmacol, 2020. 246 : p. 112228.

32. Mathew, S. and T.E. Abraham, Bioconversions of ferulic acid, an hydroxycinnamic acid. Crit Rev Microbiol, 2006. 32(3): p. 115-25. 
33. Aljawish, A., et al., Laccase mediated-synthesis of hydroxycinnamoyl-peptide from ferulic acid and carnosine. J Biotechnol, 2016. 227: p. 83-93.

34. Perez-Rodriguez, N., et al., Ferulic acid transformation into the main vanilla aroma compounds by Amycolatopsis sp. ATCC 39116. Appl Microbiol Biotechnol, 2016. 100(4): p. 1677-1689.

35. Pickett, B.D., J.B. Miller, and P.G. Ridge, Kmer-SSR: a fast and exhaustive SSR search algorithm. Bioinformatics, 2017. 33(24): p. 3922-3928.

36. Pan, Y.L., et al., [Construction of A Genetic Linkage Map in Salvia miltiorrhiza]. Zhong Yao Cai, 2016. 39(7): p. 1443-5.

\section{Tables}

Table 1. Summary statistics of the BUSCO analysis

\begin{tabular}{|lll|}
\hline Datasets & Groups $\varangle$ $\%$ & \\
\hline & Viridplantae dataset & Embryophyta dataset \\
\hline Complete BUSCOs & $386(90.8 \%)$ & $1276(79.1 \%)$ \\
\hline Complete and single-copy BUSCOs & $242(56.9 \%)$ & $816(50.6 \%)$ \\
\hline Complete and duplicated BUSCOs & $144(33.9 \%)$ & $460(28.5 \%)$ \\
\hline Fragmented BUSCOs & $37(8.7 \%)$ & $178(11.0 \%)$ \\
\hline Missing BUSCOs & $2(0.5 \%)$ & $160(9.9 \%)$ \\
\hline Total BUSCO groups searched & 425 & 1614 \\
\hline
\end{tabular}

\section{Figures}




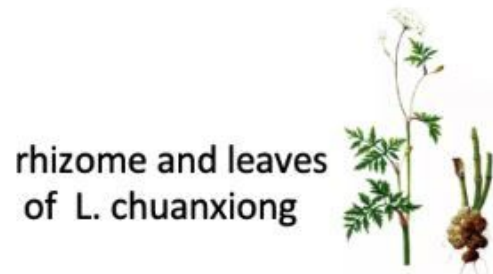

RNA extraction CTAB protocol
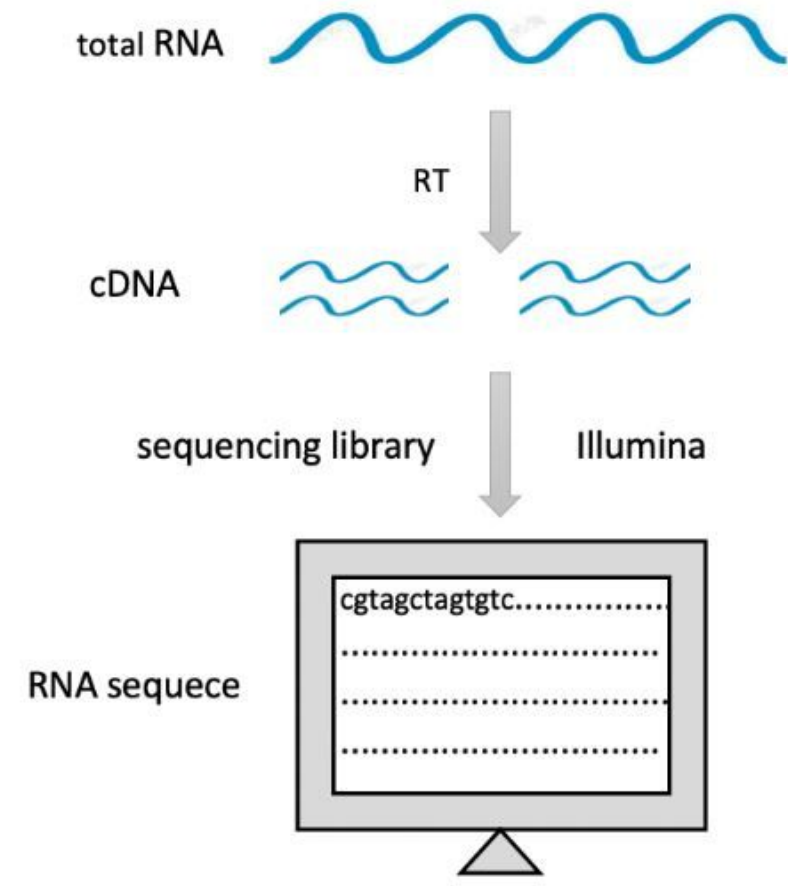

assembled using Trinity

\section{Figure 1}

Workflow diagram. RNA from Chuanxiong rhizome leaves and rhizomes was extracted and constructed into a cDNA library by reverse transcription and sequenced using Illumina HiSeq 2000. The acquired unigenes were subjected to differentially expressed gene analysis and functional annotation. 


\section{Leaf vs Rhizome}

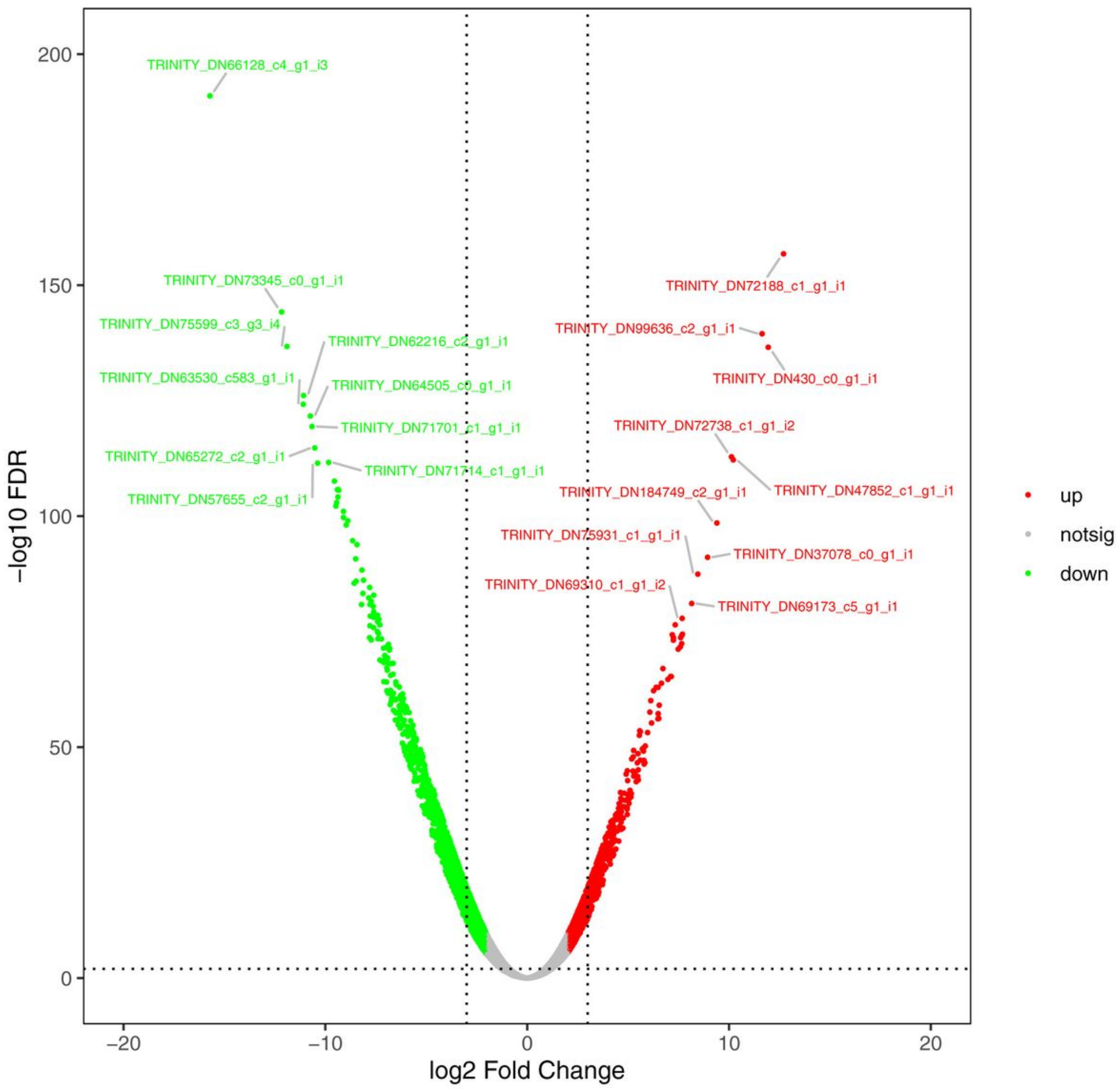

\section{Figure 2}

The horizontal coordinate represents log2 (Fold Change) and the vertical coordinate represents - $\log 10$ ( $P$ value), each point represents a gene, and the color is used to distinguish whether the gene is differentially expressed or not. Red dots represent up-regulated differentially expressed genes, while green dots represent down-regulated differentially expressed genes. 


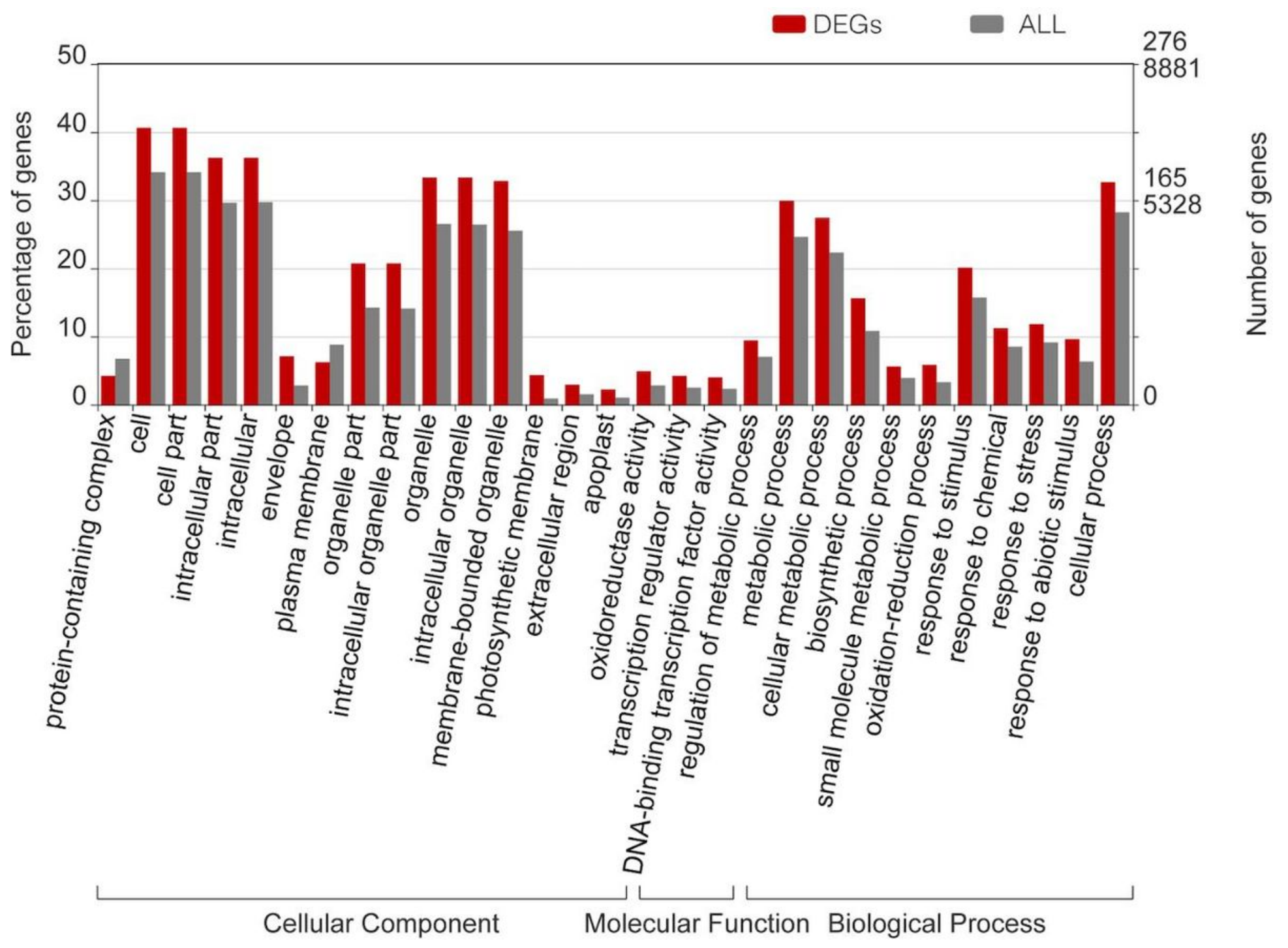

Figure 3

Summary of GO term enrichment. In the GO term analysis plot, green indicates biological processes; orange indicates cellular composition; blue indicates molecular function. The horizontal axis represents each functional category, and the vertical axis represents the number of differentially expressed genes in each functional category. 


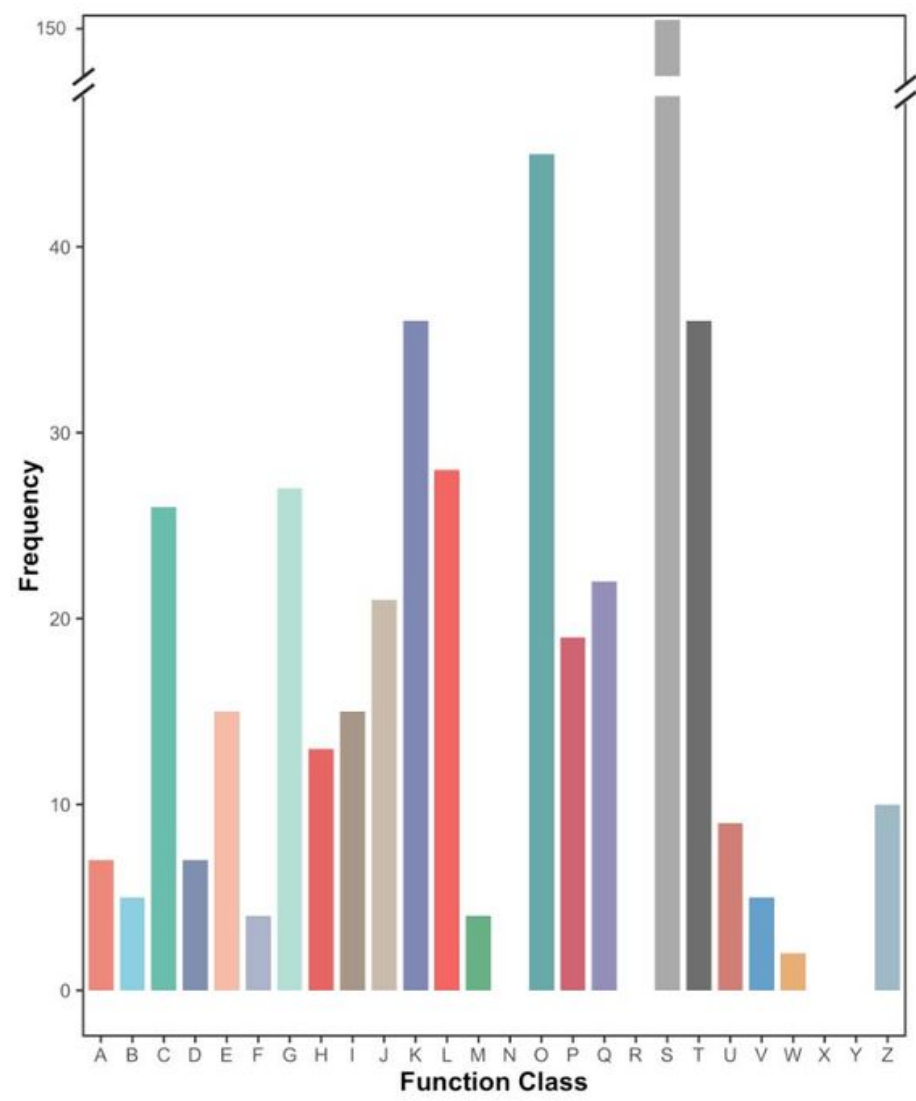

RNA processing and modification Chromatin structure and dynamics Energy production and conversion Cell cycle control, cell division, chromosome partitioning Amino acid transport and metabolism Nucleotide transport and metabolism Carbohydrate transport and metabolism Coenzyme transport and metabolism Lipid transport and metabolism Translation, ribosomal structure and biogenesis Transcription Replication, recombination and repair Cell wall/membrane/envelope biogenesis Cell motility Posttranslational modification, protein turnover, chaperones Inorganic ion transport and metabolism Secondary metabolites biosynthesis, transport and catabolism General function prediction only Function unknown Signal transduction mechanisms Intracellular trafficking, secretion, and vesicular transpor Defense mechanisms Extracellular structures Mobilome: prophages, transposons Nuclear structure Cytoskeleton

Figure 4

COG cluster. Each letter on the horizontal axis indicates a functional classification and the vertical axis indicates the number of differential genes contained in that functional classification. 


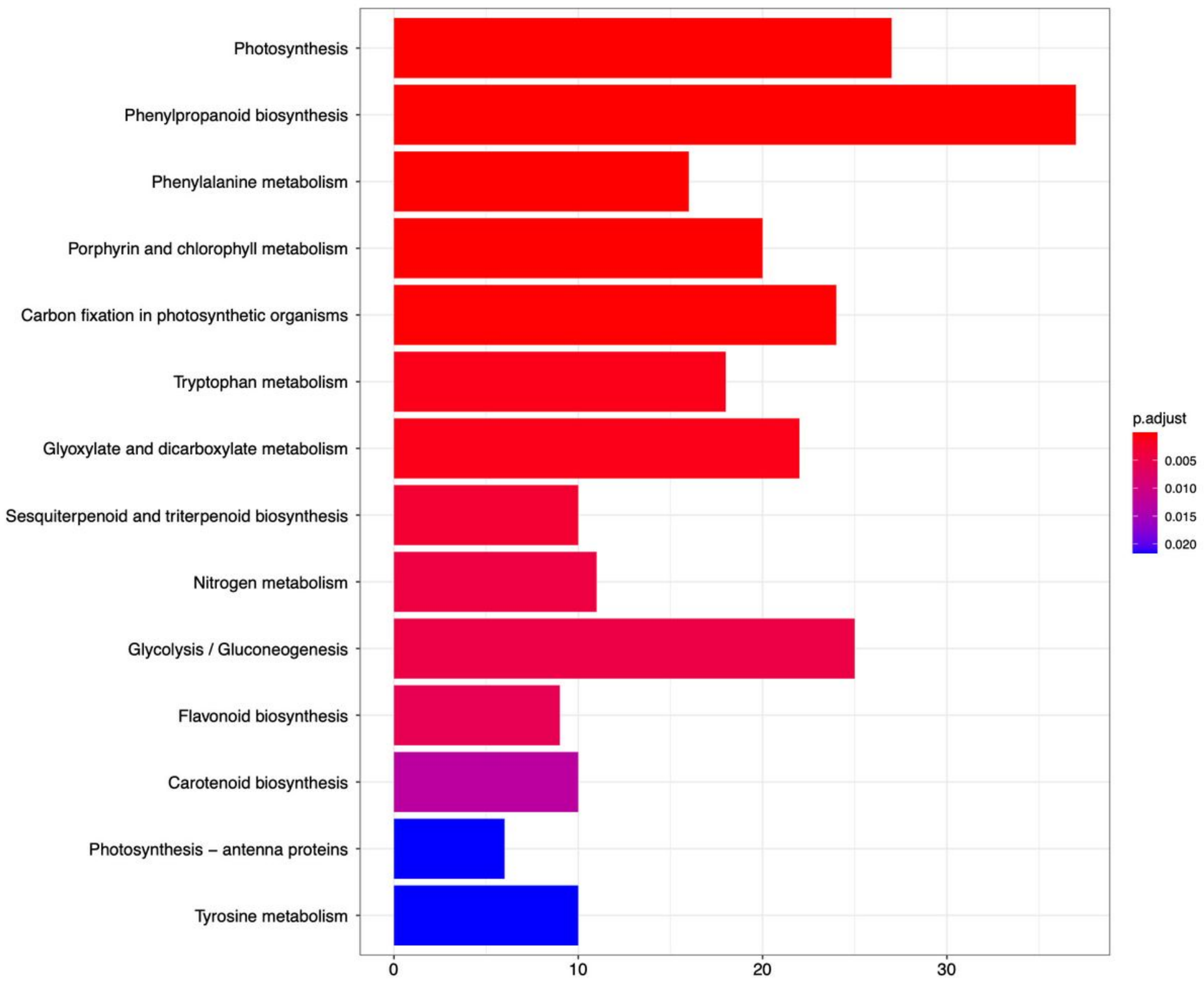

\section{Figure 5}

KEGG enrichment analysis. The vertical axis represents the type of biological pathway and the horizontal axis represents the number of differentially enriched genes in that pathway. p-value from 0.005 to 0.02 adjusted from top to bottom, the smaller the $p$-value, the more enriched the differential genes are in that pathway.

\section{Supplementary Files}

This is a list of supplementary files associated with this preprint. Click to download.

- SupplementaryData.docx 